\title{
A MOLECULAR CLOUD INTERACTING WITH THE VERTICAI FILAMENTS OF THE GALACTIC CENTER RADIO ARC
}

\author{
TOMOHARU OKA \\ Cosmic Radiation Laboratory \\ The Institute of Physical and Chemical Research (RIKEN) \\ Hirosawa 2-1, Wako, Saitama 351-01, Japan \\ TETSUO HASEGAWA \\ Institute of Astronomy, Faculty of Science, University of Tokyo \\ Osawa 2-21-1, Mitaka, Tokyo 181, Japan \\ FUMIO SATO \\ Department of Astronomy and Earth Sciences, Tokyo Gakugei University \\ Nukuikitamachi 4-1-1, Koganei, Tokyo 184, Japan
}

AND

MASATO TSUBOI AND ATSUSHI MIYAZAKI

Institute of Astrophysics and Planetary Sciences, Ibaraki University

Bunkyo 2-1-1, Mito, Ibaraki 310, Japan

\section{Introduction: Vertical Radio Filaments}

Surely the most striking radio feature in the Galactic center may be a sheaf of straight vertical filaments (VFs, e.g., Yusef-Zadeh, Morris, \& Chance 1984 ) of the radio arc. The VFs are believed to be the manifestations of strong magnetic field lines $(\geq 1 \mathrm{mG})$ which have been illuminated by some local relativistic particle source.

There are many hypotheses for production of these relativistic electrons (e.g., Serabyn \& Morris 1994). Recently, Tsuboi, Ukita, \& Handa (1997) have discovered a cloud adjacemt to the VFs in their CS maps. They found morphological evidence for the intimate interaction between the cloud and the VFs, and claimed that the interaction may activate relativistic electrons in the VFs.

\section{Molecular Gas around the Vertical Filaments}

Our ${ }^{12} \mathrm{CO} J=1-0$ and ${ }^{13} \mathrm{CO} J=1-0$ images taken with the NRO $45 \mathrm{~m}$ telescope reveal parsec-scale structure of molecular gas around the Galactic 
center radio arc (Oka et al. 1997a, 1997b, Hasegawa et al. 1997) ${ }^{1}$. We notice a compact cloud, CO 0.13-0.13, which seems to avoid the sheaf of the VFs in the plane of the sky. This is a CO counterpart of that discovered by Tsuboi, Ukita, \& Handa (1997). CO emission exhibits abrupt velocity change at the position of the VFs.
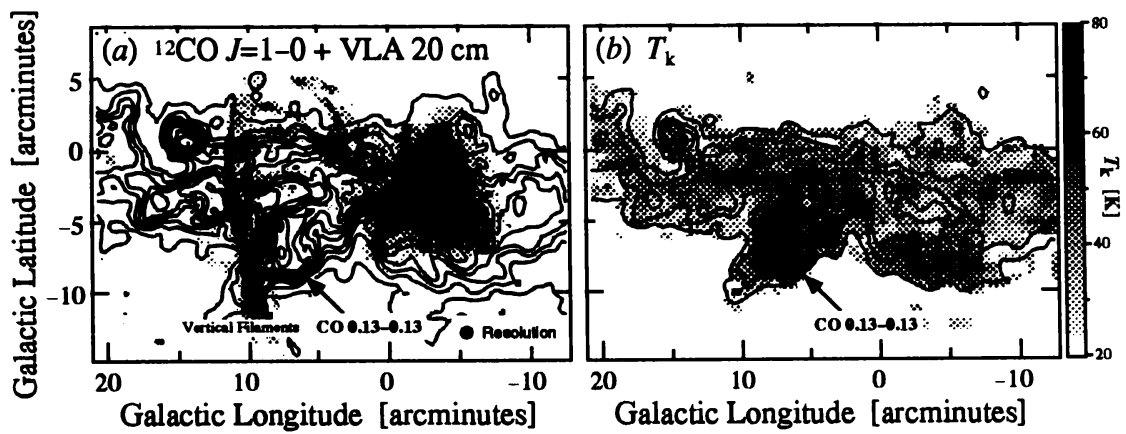

Figure 1. (a) Map of ${ }^{12} \mathrm{CO}$ emissivity integrated over $V_{\mathrm{LSR}}=30$ to $35 \mathrm{~km} \mathrm{~s}^{-1}$ (contours). Contour intervals are $10 \mathrm{~K} \mathrm{~km} \mathrm{~s}^{-1}$. VLA $20 \mathrm{~cm}$ continuum image is also shown (gray scale). (b) Map of kinetic temperature (gray scale) and ${ }^{12} \mathrm{CO}$ emissivity (contours) in the same velocity range as panel $a$ ).

An excitation analysis of ${ }^{12} \mathrm{CO}-{ }^{13} \mathrm{CO}$ correlation plot shows that gas kinetic temperature is enhanced $\left(T_{\mathrm{k}} \geq 70 \mathrm{~K}\right)$ in $\mathrm{CO} 0.13-0.13$. This lends another support for the interaction between the cloud and the VFs. Either of shock dissipation or magnetic viscosity can account for the temperature enhancement quantitatively.

We also found a large expanding shell in the other side of the VFs. The radius of the shell is $5 \mathrm{pc}$ and the expanding velocity is $25 \mathrm{~km} \mathrm{~s}^{-1}$, and hence the expansion time is $2 \times 10^{5}$ years. Kinetic energy of the shell is $\sim 5 \times$ $10^{51} \mathrm{erg}$, which corresponds to the amount converted from several $\times 10^{1-2}$ supernovae. Magnetic tubes could have been swept up by the expanding shell, and compressed by the interaction with $\mathrm{CO} 0.13-0.13$. The in teraction between the cloud and magnetic field may accelerate electrons to relativistic energies through field reconnection or induced electric field (Benford 1988).

\section{References}

Benford, G. 1988, ApJ, 333, 735

Hasegawa, T., Oka, T. Sato, F., Tsuboi, M., and Miyazaki, A. 1997, in these proceedings Oka, T., Hasegawa, T., Sato, F., Tsuboi, M., and Miyazaki, A. 1997a, ApJ submitted Oka, T., Hasegawa, T., Sato, F., Tsuboi, M., and Miyazaki, A. 1997b, ApJS submitted Serabyn, E. \& Morris, M. 1994, ApJ, 424, L91

Tsuboi, M., Ukita, N., \& Handa, T. 1997, ApJ, 481, 263

Yusef-Zadeh, F., Morris, M., \& Chance, D. 1984, Nature, 310, 557

\footnotetext{
${ }^{1}$ This work is based on the Nobeyama Radio Observatory (NRO) $45 \mathrm{~m}$ telescope key program: 'A Large-Scale CO Imaging of the Galactic Center'.
} 